\title{
Editorial
}

Alfred Königsrainer* and Beate Rau

\section{Cytoreductive Surgery (CRS) and Hyperthermic IntraPeritoneal Chemotherapy (HIPEC): don't throw the baby out with the bathwater}

https://doi.org/10.1515/pap-2018-0131

Results of the French randomized PRODIGE 7 trial presented this June at ASCO meeting show that adding Hyperthermic IntraPeritoneal Chemotherapy (HIPEC) with oxalipatin to optimal cytoreductive surgery does not improve survival in peritoneal metastasis of colorectal origin [1]. A high dose of oxaliplatin $\left(460 \mathrm{mg} / \mathrm{m}^{2}\right.$ body surface, equivalent to five times an intravenous dose) was applied and there was an increased risk of long-term complications. Clearly, given the lack of survival benefit and the increased risk of postoperative complications with HIPEC, incorporating intraperitoneal oxaliplatin-based chemotherapy into the standard treatment regimen for peritoneal metastatic colorectal cancer should be reconsidered.

These results were object of intensive discussions among HIPEC surgeons during the last meeting of the Peritoneal Surface Oncology Group International (PSOGI) in Paris on September 9th-11th, 2018. In particular, following questions were raised:

Is HIPEC beneficial for a subgroup of patients with a midrange peritoneal cancer index?

Can people with a low peritoneal cancer index forgo HIPEC?

Is 30 minutes of HIPEC long enough?

Do patients with a high index not benefit from either surgery or HIPEC?

Which drug should be used in the future, if any?

Is systemic chemotherapy necessary compared with CRS ?

It was also noted that the results of the PRODIGE 7 trial have not yet been object of a full, peer-reviewed publication. Methodological standards require that a full, peer-reviewed publication should be awaited to answer the questions above. Whereas it appears legitimate to discuss the results presented at the ASCO meeting, it is premature to change clinical practice, therapy guidelines or reimbursement policies before the full publication is available.
For many physicians, it might also appear premature to conclude at this stage that "this is the end of CRS and HIPEC for peritoneal metastasis of colorectal origin" [2]. When the favorable is rejected along with the unfavorable, the risk is that patients might be discouraged or prevented to receive the best possible treatment.

In fact, results of the PRODIGE-7 trial show an overall survival of 41 months after cytoreductive surgery alone. This long survival was not expected by the initiators of the study when they submitted their protocol. This is an unequivocal and positive finding for surgeons specialized in therapy of peritoneal metastasis and for their patients. Thus, results of the trial strengthen - and not weaken - the need for patients with isolated peritoneal metastasis of colorectal origin to be referred to a tertiary center for evaluation of the possibility of complete surgical cytoreduction. PRODIGE-7 confirms that this therapy offers the best chances of survival in selected patients with isolated peritoneal metastasis with an overall survival which is by far not reached by systemic chemotherapy alone (16 months) [3].

The PRODIGE-7 trial has been performed in a welldefined cohort of patients meeting precise inclusion and exclusion criteria. In particular, all patients had colorectal cancer. Thus, extrapolation of the absence of additional survival after HIPEC in colorectal cancer to other primary tumors is not permissible. This assumption is confirmed by the recent publication of the positive results of another randomized trial in ovarian cancer showing a beneficial effect of HIPEC on survival, as compared to surgery alone [4].

In the PRODIGE 7 trial, the drug used was oxaliplatin. However, outside Europe and in particular in the UK and in the USA, the most common drug administered as HIPEC in colorectal cancer is mitomycin C (MMC), not oxaliplatin [5]. The potential survival advantage of administering MMC as HIPEC in addition to CRS was not investigated in a proper randomized controlled trial. Thus, at the present time and on the basis of data available, it is not possible to conclude that HIPEC with MMC has no effect in selected patients with peritoneal metastasis of colorectal origin. 
For almost 20 years, we know that oxaliplatin is a drug with verified efficacy in colorectal cancer [6]. The negative results of the PRODIGE 7 trial cannot be explained by the drug chosen, but rather by pharmacological limitations of HIPEC as a drug delivery system, in particular limited tissue penetration [7]. Experimentally, Pressurized IntraPeritoneal Aerosol Chemotherapy (PIPAC) achieved better tissue penetration of oxaliplatin than HIPEC in organoids [8]. This superior efficacy of PIPAC in delivering oxaliplatin has been confirmed in the clinical setting, where PIPAC was able to induce objective radiological and histological regression of peritoneal metastases of colorectal origin [9]. For this reason it is not possible to extrapolate the results of oxaliplatin applied as HIPEC to other intraperitoneal drug delivery techniques.

The negative results of the PRODIGE-7 trial should be discussed and might change clinical practice in the near future. However, oncologists should not throw the baby out with the bathwater. We are confident that they will not since they are used to such failures. For example, immune therapies, including checkpoint antibodies and engineered $\mathrm{T}$ cell infusions, have shown little efficacy to date in pancreatic cancer. However, major clinical efforts, justified by preclinical models, are still ongoing to address multiple immune vulnerabilities in this indication [10].

Thus, is not because of a negative trial in peritoneal metastasis of colorectal origin that HIPEC should be abandoned. It has to be remembered that peritoneal metastasis remains a lethal disease and that available therapies, including modern combination chemotherapy, are not effective enough to keep patients alive. In the legitimate interest of patients with peritoneal metastasis, funding bodies should further support actively research in the field. More research is needed to determine which patients are still benefiting from receiving HIPEC or other forms of intraperitoneal drug delivery in addition to cytoreductive surgery.

Author contributions: All the authors have accepted responsibility for the entire content of this submitted manuscript and approved submission.

Research funding: None declared.

Employment or leadership: None declared.

Honorarium: None declared.

\section{References}

1. Quenet F, Elias D, Roca L, Goere D, Ghouti L, Pocard M, et al. A UNICANCER phase III trial of hyperthermic intra-peritoneal chemotherapy (HIPEC) for colorectal peritoneal carcinomatosis (PC): PRODIGE 7. J Clin Oncol. 2018;36:abstr LBA3503.

2. Evrard S. Autopsy of an expert consensus: end of hyperthermic intraperitoneal chemotherapy in colorectal carcinomatosis. Eur J Surg Oncol. pii: S0748-7983(18)31270-8. Epub ahead of print 2018. DOI: 10.1016/j.ejso.2018.07.061.

3. Franko J, Shi Q, Meyers JP, Maughan TS, Adams RA, Seymour MT, et al. Prognosis of patients with peritoneal metastatic colorectal cancer given systemic therapy: an analysis of individual patient data from prospective randomised trials from the analysis and research in cancers of the digestive system (ARCAD) database. Lancet Oncol. 2016;17:1709-19.

4. Van Driel WJ, Koole SN, Sikorska K, Schagen van Leeuwen JH, Schreuder HWR, Hermans RHM, et al. Hyperthermic intraperitoneal chemotherapy in ovarian cancer. $\mathrm{N}$ Engl J Med. 2018;378:230-40.

5. Van der Speeten K, Lemoine L, Sugarbaker P. Overview of the optimal perioperative intraperitoneal chemotherapy regimens used in current clinical practice. Pleura Peritoneum. 2017;2:63-72.

6. de Gramont A, Figer A, Seymour M, Homerin M, Hmissi A, Cassidy J, et al. Leucovorin and fluorouracil with or without oxaliplatin as first-line treatment in advanced colorectal cancer. J Clin Oncol. 2000;18:2938-47.

7. Lagast N, Carlier C, Ceelen WP. Pharmacokinetics and tissue transport of intraperitoneal chemotherapy. Surg Oncol Clin N Am. 2018;27:477-94.

8. Eveno C, Haidara A, Ali I, Pimpie C, Mirshahi M, Pocard M. Experimental pharmacokinetics evaluation of chemotherapy delivery by PIPAC for colon cancer: first evidence for efficacy. Pleura Peritoneum. 2017;2:103-9.

9. Demtröder C, Solass W, Zieren J, Strumberg D, Giger-Pabst U, Reymond MA. Pressurized intraperitoneal aerosol chemotherapy with oxaliplatin in colorectal peritoneal metastasis. Colorectal Dis. 2016;18:364-71.

10. Morrison AH, Byrne KT, Vonderheide RH. Immunotherapy and prevention of pancreatic cancer. Trends Cancer. 2018;4:418-28.

Alfred Königsrainer, Klinik für Allgemeine, Viszteral- und Transplantationschirurgie, Universitätsklinikum Tübingen, HoppeSeyler Str. 3 72076, Tübingen, Germany, E-mail: alfred.koenigsrainer@med.uni-tuebingen.de

Beate Rau, Department of Surgery Charité, Universitätsmedizin, Berlin, Germany, E-mail: beate.rau@charite.de 